\title{
ASSISTÊNCIA FARMACÊUTICA NA GESTÃO DE MEDICAÇÃO DA SAÚDE MENTAL
}

\author{
Aline Ferreira Campos Correia \\ Ivânia Vera \\ Roselma Lucchese \\ Kamylla Guedes de Sena \\ Roberta Almeida Elias \\ (Universidade Federal de Goiás, Regional Catalão)
}

\begin{abstract}
Resumo
O tratamento da esquizofrenia é realizado com o uso de fármacos antipsicóticos atípicos, considerado um tratamento de elevado custo sendo subsidiado pelo Sistema Único de Saúde. O presente estudo tem como objetivo apresentar a assistência farmacêutica na gestão de custos dos pacientes esquizofrênicos que fazem uso de antipsicóticos atípicos dispensados pelo programa de medicamentos excepcionais do Governo Federal no decorrer de seu tratamento. Trata-se de uma revisão bibliográfica. Verificou-se que pacientes do sexo masculino em comparação ao feminino apresentam um tratamento com custo mais elevado. Percebe-se uma eficiente assistência farmacêutica na gestão de gastos públicos provenientes do fornecimento de medicamentos excepcionais a pacientes com esquizofrenia, devido promoverem um melhor prognostico em comparação aos típicos.
\end{abstract}

Palavras-chave: antipsicóticos; esquizofrenia; assistência farmacêutica.

\section{Abstract \\ Pharmaceutical Care in Management of Mental Health Medication}

The treatment of schizophrenia is performed with the use of atypical antipsychotic drugs, considered a high cost treatment being subsidized by the Unified Health System. The present study aims to present the pharmaceutical assistance in the cost management of schizophrenic patients who use antipsychotics atypical drugs dispensed by the Federal Government's exceptional medicines program during its treatment. This is a bibliographical review. It was found that male patients in comparison to the female present a treatment with higher cost. An efficient pharmaceutical assistance in the management of public expenditures from the supply of exceptional medicines to patients with schizophrenia is perceived, because they promote a better prognosis in comparison with the typical ones.

Key words: antipsychotic agents; schizophrenia; pharmaceutical services. 


\section{Introdução}

Os antipsicóticos atípicos representam a base para o tratamento de pessoas com transtornos mentais, em especial a esquizofrenia (SCZ) (Rang et al., 2012). Este tratamento farmacológico é considerado de alto custo, no entanto é subsidiado pelo Sistema Único de Saúde (SUS) (Brasil, 2017). De acordo com dados apresentados pelo DATASUS indicam que, o gasto com medicamentos de alto custo foi, em média, $2 \%$ do gasto federal total com saúde no período de 2000 a 2004 (Silva, Guerra Júnior, Acúrcio, Cherchiglia \& Andrade, 2011).

A SCZ é um transtorno psiquiátrico caracterizado pelo surgimento de sintomas psicóticos positivos da doença (delírios e alucinações), os quais posteriormente evolucionam para um estado crônico, caracterizado pelos sintomas negativos da doença como acatisia, apatia, seguidos ou não por novos episódios de delírios e alucinações, não transmissível. Esse transtorno é classificado através do Código Internacional de Doenças, entre a denominação de F20.0 a F29 (Who, 1997; Who, 2017).

Devido a demanda do fornecimento de medicamentos para tratamentos de doenças crônicas, ou seja, "Medicamentos de Dispensação Excepcional" foi criado em 1982 o Componente Especializado da
Assistência Farmacêutica (CEAF). Esta iniciativa caracteriza-se como uma estratégia da Política de Assistência Farmacêutica, objetivando disponibilizar medicamentos para a terapêutica de doenças específicas e garantir a integralidade da farmacoterapia, no nível ambulatorial, cujos parâmetros de cuidado estão deliberados em Protocolos Clínicos e Diretrizes Terapêuticas (PDCT) (Brasil, 2009).

Os medicamentos que fazem parte do CEAF foram regulamentados pela Portaria do Gabinete do Ministro do Ministério da Saúde (GM/MS) nº 2.981, de 26 de novembro de 2009 e, a partir desta data se solidificou como uma relevante estratégia para a aquisição de fármacos no SUS (Brasil, 2009).

Sua particularidade é a segurança da plenitude terapêutica e medicamentosa para todas as doenças enquadradas no CEAF. Assim, os compostos farmacológicos e outros procedimentos em saúde relevantes para assegurar a integralidade são deliberados nos Protocolos Clínicos e Diretrizes Terapêuticas (PCDT) através dos distintos traçados de atenção (Brasil, 2009).

É importante enfatizar que o acesso aos compostos farmacológicos de alto custo, consiste em direito à saúde admitido pela Organização Mundial da Saúde (OMS) e foi um indicador de avanço do país incluso no Plano Estratégico de Médio Prazo da 
OMS para 2008-2013 (Ramagem \& Ruales, 2008). O exemplo de gestão da tabela de dispensação de medicamentos de alto custo precisaria seguir os princípios da alocação estratégica de recursos recomendado pela OMS, ou seja, "fazer o que é preconizado, de maneira apropriada e no lugar adequado" (Brasil, 2005).

A participação e aquisição de medicamentos via CEAF, possui algumas etapas, para a abertura do processo, o indivíduo ou seu responsável deve solicitar o Laudo de Solicitação de Medicamentos (LME) em uma unidade das Farmácias de Medicamentos Especializados (FME), com a apresentação de documentos do paciente conforme o Protocolos Clínicos e Diretrizes Terapêuticas do Ministério da Saúde. Um LME tem validade de três meses, é específico para cada doença (CID) (Brasil, 2017).

A farmacoterapia dos antipsicóticos utilizados na dispensação de alto custo para esquizofrenia também chamados de segunda geração ou atípicos é preconizada no Protocolo Clínico e Diretrizes Terapêuticas- Esquizofrenia - Ministério da Saúde (PCDT/MS) são eles: risperidona apresentados como comprimidos na dosagem 1, 2 e $3 \mathrm{mg}$ ), quetiapina comprimidos de 25, 100, 200 e $300 \mathrm{mg}$-, ziprasidona - cápsulas de 40 e 80 mg - e clozapina (comprimidos de 25 e $100 \mathrm{mg}$ ) (Brasil, 2013).
A renovação do $\mathrm{LME}$, depende de consulta e prescrição médica atualizada e, nos esquizofrênicos exige-se exames complementares de sangue (hemograma, contagem de leucócitos e plaquetas devido ao uso de clozapina, citopenia caracterizada por leucopenia ou por plaquetopenia), perfil lipídico, escala breve de avaliação psiquiátrica (brief psychiatric rating scale BPRS) e medidas antropométricas descritos no PDCT/MS, para efeito de monitorização (Brasil, 2017).

A BPRS deve ser preenchida pelo médico psiquiatra e consiste em avaliar a gravidade da doença em relação à semana anterior vivenciada e o grau de mudança avalia a alteração da gravidade da desordem entre dois espaços de tempo. Sendo que cada categoria dispõe de cinco classificações distintas para analisar sintomas positivos, negativos, depressivos, cognitivos e globais e sua versão é validada para a população brasileira (Lima et al., 2007).

Para estimar o custo do tratamento da esquizofrenia com os antipsicóticos atípicos é importante considerar o tempo do tratamento, dosagem do medicamento, idade e expectativa de vida do paciente (Lindner, Marasciulo, Farias \& Grohs, 2009). A literatura postula que a expectativa de vida do esquizofrênico é reduzida em 10 a 25 anos em relação a população em geral 
(Laursen, Munk-Olsen \& Vestergaard, 2012).

Em decorrência da redução na expectativa de vida, os pacientes esquizofrênicos não conseguem vivenciar uma evolução na qualidade de vida, devido a vulnerabilidade a outras patologias as quais estão sujeitos (Laursen, Munk-Olsen \& Vestergaard, 2012).

Desse modo, este estudo tem por objetivo apresentar a assistência farmacêutica na gestão de custos dos pacientes esquizofrênicos que fazem uso de antipsicóticos atípicos dispensados pelo programa de medicamentos excepcionais (medicamentos de alto custo) do Governo Federal no decorrer de seu tratamento.

\section{Metodologia}

Esse estudo é uma revisão bibliográfica que se amplifica no desenvolvimento de etapas, que para se desenvolver resultam de princípios, tais como a natureza do problema, o nível de informação que o pesquisador dispõe sobre a temática, o grau de precisão que se espera aprofundar a pesquisa, entre outros aspectos (Gil, 2009).

O levantamento dos dados da pesquisa foi realizado usando os seguintes descritores de forma combinada: esquizofrenia, gestão de medicação, assistência farmacêutica, saúde mental, sistema único de saúde, nas bases de dados da Literatura Latino - Americana e do Caribe em Ciências da Saúde (LILACS); Medical Literature Analysis and Retrieval Sistem online (MedLine), Biblioteca Nacional de Medicina (Pubmed) e Scientific Electronic Library Online (SciELO), no meses de junho, julho e agosto de 2017.

Os critérios de inclusão no estudo foram: artigos completos, originais, dos últimos anos; em língua inglesa e portuguesa, que abordassem o assunto proposto de 2013 a 2017. Foram excluídos os artigos que se repetiram nas bases de dados.

Nesta pesquisa realizou-se o cálculo dos valores dos medicamentos dispensados nos programas de medicamentos excepcionais (alto custo) em relação à idade de diagnóstico do paciente (sexos: feminino $=25$ a 35 anos, média $=30$ anos e masculino $=15$ a 25 anos, média $=20$ anos) (APA, 2014) e expectativa de vida do paciente esquizofrênico relatada que é reduzida em 10 a 24 anos, média de 17,5 (sexos: feminino $=79,1-17,5=61,6 \quad \mathrm{e}$ masculino $=71,9-17,5=54,4 \quad$ (Laursen, Munk-Olsen \& Vestergaard, 2012).

Portanto utilizando os valores encontrados na expectativa de vida (EV) do paciente esquizofrênico reduzidos da média de idade de diagnóstico (ID) foi obtido pelo cálculo: $\quad(\mathrm{EVF}=61,6-30 \mathrm{IDF}=31,6) \quad \mathrm{e}$ $(E V M=54,4-20 I D M=34,4)$. Para realização 
dos cálculos utilizou- se os medicamentos de referência, ou seja, um produto registrado no órgão federal responsável pela vigilância sanitária e comercializado no País, cuja eficácia, segurança e qualidade foram comprovadas cientificamente junto ao órgão competente, por ocasião do registro (Anvisa, 2017a).

No mercado farmacêutico existem diversos fabricantes de antipsicóticos atípicos genéricos e similares nas dosagens padrões, porém para os cálculos utilizou- se os medicamentos de referência, ou seja, um produto inovador registrado no órgão federal responsável pela vigilância sanitária e comercializado no País, cuja eficácia, segurança e qualidade foram comprovadas cientificamente junto ao órgão federal competente, por ocasião do registro, o primeiro a ser fabricado no mercado (Anvisa, 2017a).

Os medicamentos subsidiados pelo governo federal fazem parte de uma lista de preços individualizada que contém o maior custo no qual os responsáveis pela Administração Pública realizam obtenção dos medicamentos dos laboratórios, distribuidores, farmácias e drogarias (Anvisa, 2017b).
Em virtude do elevado custo comercial dos antipsicóticos atípicos para os pacientes, foi realizada a verificação de valores dos medicamentos antipsicóticos atípicos conforme a Figura 1. Constatou-se que o custo de um tratamento para pacientes esquizofrênicos é superior para pacientes do sexo masculino, visto que a idade do cálculo de uso da medicação para o homem foi de 34,4 anos enquanto para as pacientes do sexo feminino foi de 31,6 anos.

O medicamento com o custo mais alto no tratamento foi o Hemifumataro de quetiapina/Seroquel para pacientes do sexo masculino com dosagem máxima de $600 \mathrm{mg} / \mathrm{dia}$, quando utilizado na prescrição de caixa de 100mg. Em contrapartida, o custo de tratamento com o valor mais baixo foi o medicamento Risperidona/Risperdal, ao ser utilizado para pacientes do sexo feminino com dosagem mínima de $3 \mathrm{mg} / \mathrm{dia}$, quando utilizado na prescrição de caixa de $1 \mathrm{mg}$.

Em um estudo desenvolvido no México, evidenciou que o tratamento medicamentoso consiste na intervenção para a SCZ de maior custo. E revela ainda que a junção terapêutica de antipsicóticos atípicos e terapia psicológica acentuam os gastos, porém apresenta uma alta eficiência na condução terapêutica (Lara-Muñoz et al., 2010).

Dados do Instituto Brasileiro de Geografia e Estatística - IBGE (2016) 
revelam que o Rendimento nominal domiciliar per capita da população brasileira no ano de 2016 foi de $\mathrm{R} \$$ 1.226,00, sendo este valor convertido em dólar de \$386,63. Desta forma, o fornecimento da medicação por parte do Governo Federal para pacientes com esquizofrenia é de vital importância, pois mesmo comprometendo a sua renda total, não seria possível para um brasileiro custear o tratamento com a medicação de menor valor econômico apresentando na Figura 1.

Corroborando com os resultados encontrados a utilização do Hemifumarato de quetiapina, representa uma estratégia eficaz no tratamento da SCZ, quanto os fármacos de referência tradicional. Foram constatados poucos efeitos adversos, sendo os mais comuns a tontura, a cefaleia e a sonolência (Oliveira, 2000).
Um estudo observou que o gênero masculino, em relação ao cálculo do tempo de tratamento se sobressaiu ao gênero feminino. Isso pode ser atribuído pelo prolongamento da presença de polifarmácia (uso concomitante de 5 ou mais medicamentos ao dia) e a idade de início da doença precoce que se constitui também em fatores associados à refratariedade clínica, devido a interrupção e não adesão a farmacoterapia (Cezaretto et al., 2014).

Considerando os dados apresentados nessa pesquisa, sobre os valores referentes ao custo da risperidona, que apresenta ser o medicamento de menor custo, evidencia-se a eficácia terapêutica desse fármaco de longa duração, sendo a formulação em questão aceita como a melhor escolha clínica na terapêutica de manutenção da esquizofrenia, em virtude do seu baixo índice de reincidência e acréscimo na qualidade de vida do paciente (Nascimento, Lomez \& Gaspar, 2014). 
ASSISTÊNCIA FARMACÊUTICA NA GESTÃO DE MEDICAÇÃO DA SAÚDE MENTAL

Figura 1

Estimativa dos Custos dos Antipsicóticos Atípicos segundo Sexo e Idade, 2017

\begin{tabular}{|c|c|c|c|c|c|c|c|}
\hline \multirow{4}{*}{ Medicamento Referência/ dose unitária } & \multirow{4}{*}{$\begin{array}{l}\text { Custo (USS) } \\
\text { dosagem unitária }\end{array}$} & \multirow{4}{*}{$\begin{array}{l}\text { Custo (US\$) } \\
\text { dosagem } \\
\text { mínima/ano }\end{array}$} & \multirow{4}{*}{$\begin{array}{l}\text { Custo (US\$) } \\
\text { dosagem } \\
\text { máxima/ano }\end{array}$} & \multicolumn{4}{|c|}{ Custo do medicamento por tempo de vida" } \\
\hline & & & & & & & \\
\hline & & & & \multirow{2}{*}{\multicolumn{2}{|c|}{$\begin{array}{l}\text { Masculino - } 34,4 \text { anos }^{2} \\
\text { Dosagem }\end{array}$}} & \multirow{2}{*}{\multicolumn{2}{|c|}{$\begin{array}{l}\text { Feminino- } 31,6 \text { anos } \\
\text { Dosagem }\end{array}$}} \\
\hline & & & & & & & \\
\hline Cloridrato de ziprasidona /Geodon & & 80mg/dia/ano & $160 \mathrm{mg} / \mathrm{dia} / \mathrm{ano}$ & mínima & máxima & mínima & máxima \\
\hline $40 \mathrm{mg}$ & 2,17 & $1.562,16$ & $3.124,31$ & $53.738,20$ & $107.476,40$ & $49.364,16$ & $98.728,33$ \\
\hline $80 \mathrm{mg}$ & 3,61 & $1.299,91$ & $2.599,81$ & $44.716,75$ & $89.433,49$ & $41.077,01$ & $82.154,02$ \\
\hline Clozapina/Leponex & & $300 \mathrm{mg} / \mathrm{dia} /$ ano & $800 \mathrm{mg} / \mathrm{dia} / \mathrm{ano}$ & & & & \\
\hline $25 \mathrm{mg}$ & 0,35 & $1.512,20$ & $4.032,54$ & $52.019,83$ & $138.719,55$ & $47.785,66$ & $127.428,42$ \\
\hline $100 \mathrm{mg}$ & 1,43 & $1.549,67$ & $4.132,45$ & $53.308,61$ & $142.156,29$ & $48.969,54$ & $130.585,43$ \\
\hline \multicolumn{8}{|l|}{ Hemifumataro de quetiapina/ } \\
\hline Seroquel & & $300 \mathrm{mg} / \mathrm{dia} / \mathrm{ano}$ & $600 \mathrm{mg} / \mathrm{dia} / \mathrm{ano}$ & & & & \\
\hline $25 \mathrm{mg}$ & 0,67 & $2.874,55$ & $5.749,10$ & $98.884,54$ & $197.769,08$ & $90.835,80$ & $181.671,60$ \\
\hline $100 \mathrm{mg}$ & 2,21 & $2.390,92$ & $4.781,84$ & $82.247,57$ & $164.495,14$ & $75.553,00$ & $151.106,00$ \\
\hline $200 \mathrm{mg}$ & 3,81 & - & $4.110,88$ & - & $141.414,27$ & - & $129.903,80$ \\
\hline $300 \mathrm{mg}$ & 5,83 & $1.614,38$ & $3.228,76$ & $55.534,68$ & $111.069,37$ & $51.014,42$ & $102.028,84$ \\
\hline Olanzapina/Olanzapina & & $10 \mathrm{mg} / \mathrm{dia} / \mathrm{ano}$ & $20 \mathrm{mg} / \mathrm{dia} / \mathrm{ano}$ & & & & \\
\hline $5 \mathrm{mg}$ & 2,87 & $2.063,95$ & $4.127,91$ & $71.000,04$ & $142.000,08$ & $65.220,96$ & $130.441,93$ \\
\hline $10 \mathrm{mg}$ & 5,74 & $2.065,09$ & $4.130,18$ & $71.039,09$ & $142.078,18$ & $65.256,84$ & $130.513,68$ \\
\hline Risperidona/Risperdal & & $3 \mathrm{mg} / \mathrm{dia} / \mathrm{ano}$ & $6 \mathrm{mg} / \mathrm{dia} / \mathrm{ano}$ & & & & \\
\hline $1 \mathrm{mg}$ & 0,98 & $1.062,63$ & $2.125,26$ & $36.554,47$ & $73.108,95$ & $33.579,11$ & $67.158,22$ \\
\hline $2 \mathrm{mg}$ & 2,04 & - & $2.207,00$ & - & $75.920,83$ & - & $69.741,23$ \\
\hline $3 \mathrm{mg}$ & 3,04 & $1.095,55$ & $2.191,11$ & $37.687,04$ & $75.374,08$ & $34.619,49$ & $69.238,98$ \\
\hline
\end{tabular}

"Cotação do USS (Dólar) = 3,171 R\$ (Real). Disponivel em: hittps://economia.uol.com_br/cotacoes/cambio/dolar-comercial-estados-unidos/. Acesso em: 30/08/2017

b Conforme proposto pela literatura (Laursen et al., 2012).

Idade de diagnóstico do paciente esquizofrêncio (APA, 2014) 
Assim, pode-se demonstrar a relevância da dispensação de medicamentos de excepcionais de maneira sistematizada e integralizada para pacientes esquizofrênicos, pontuando fatores como a adesão ao tratamento, sociabilidade, renda, dificuldade de empregabilidade em decorrência das conturbações motoras e cognitivas e relacionais no ambiente de trabalho (Chen, Mccombs \& Park, 2008; Sales \& Monteiro, 2016).

\section{Considerações Finais}

Em relação à assistência farmacêutica na gestão de custos oriundos das medicações excepcionais pelo Governo Federal no decorrer do tratamento do paciente com esquizofrenia, mostra-se efetivo, uma vez que o uso de antipsicóticos atípicos promove um melhor prognóstico em comparação aos típicos.

A assistência farmacêutica não está limitada apenas à fabricação e dispensação de medicamentos, mas abrange um conjunto de processos imprescindíveis à promoção, prevenção e recuperação da saúde, individual e coletiva, centralizado nas medicações, com vistas à adequada na utilização de recursos, na expectativa de aumentar a qualidade de atenção à saúde mental.

Sendo assim, é indispensável apoiar novas pesquisas científicas que atuem no processo de avaliar a aplicação dos recursos de aquisição dos medicamentos e serviços de saúde direcionados a pacientes esquizofrênicos, em decorrência do elevado custo econômico 


\section{Referências}

Anvisa. Agência Nacional de Vigilância Sanitária (2017a). Regulação de produtos. Disponível em: http://portal.anvisa.gov.br/registros-eautorizacoes/medicamentos/produtos/medicamentos-de-referencia/informacoes-gerais. Acesso em: 12 jun. 2017a.

Anvisa. Agência Nacional de Vigilância Sanitária (2017b). Consulta de lista de preço de medicamento. Disponível em: http://portal.anvisa.gov.br/consulta-lista-de-preco-demedicamento. Acesso em: 12 jun. $2017 \mathrm{~b}$.

Brasil. Ministério da Saúde (2017). Central de Medicamentos de Alto Custo (CMAC). Disponível em: http://www.saude.go.gov.br/page/77/central-de-medicamentos-de-altocusto-cmac. Acesso em: 12 jun. 2017.

Brasil. Ministério da Saúde (2005). $1^{a}$ Conferência Nacional de Medicamentos e Assistência Farmacêutica. Disponível em: http://conselho.saude.gov.br/biblioteca/Relatorios/confer_nacional_de\%20medicamentos. pdf. Acesso em: 12 jun. 2017.

Brasil. Portaria $\mathrm{n}^{\circ} 364$, de 9 de abril de 2013, estabelece o protocolo clinico e diretrizes terapêuticas para a esquizofrenia (2013). Diário Oficial da União, Brasília, DF, 9 abr. 2013. Disponível em: http://bvsms.saude.gov.br/bvs/saudelegis/sas/2013/prt0364_09_04_2013.html._Acesso em: 12 jun. 2017

Brasil. Portaria n 2981, de 26 de novembro de 2009, estabelece as normas de execução e de financiamento da Assistência Farmacêutica na Atenção Básica (2009). Diário Oficial da União, Brasília, DF, 26 nov. 2009. Disponível em: http://189.28.128.100/dab/docs/legislacao/portaria2982_26_11_09.pdf. Acesso em: 12 jun. 2017.

Cezaretto, M., Silva, E. F. D. S. F., Ambrizzi, A., Biase, V. E. D. D., Silva, E. D. F., Cruz, E. M. T. N. D., ... \& Araújo Filho, G. M. D. (2014). Clinical and sociodemographic profile of patients with refractory schizophrenia treated in a tertiary center. Jornal Brasileiro de Psiquiatria, 63(3), 185-190. DOI 10.1590/0047-2085000000024.

Chen, L., McCombs, J. S., \& Park, J. (2008). Duration of antipsychotic drug therapy in realworld practice: a comparison with CATIE trial results. Value in Health, 11(3), 487-496. DOI 10.1111/j.1524-4733.2007.00262.x.

Gil, A. C. (2009). Como elaborar projetos de pesquisa. São Paulo: Atlas, 2002. Métodos e técnicas de pesquisa social, 6, 22-23. 
IBGE. Instituto Brasileiro de Geografia e Estatística (2016). IBGE divulga o rendimento domiciliar per capita 2016. Disponível em: $\mathrm{ftp}$ ///ftp.ibge.gov.br/Trabalho_e_Rendimento/Pesquisa_Nacional_por_Amostra_de_Domi cilios_continua/Renda_domiciliar_per_capita/Renda_domiciliar_per_capita_2016.pdf. Acesso em: 12 jun. 2017.

Lara-Muñoz, M.C., Garcia, R.R., Orozco, R., Mendez, T.S., Medina-Mora, E., Chisholm, D. (2010). Estudio de costo-efectividad del tratamiento de la esquizofrenia en México. Revista Salud Mental, 33, 211-218.

Lahon, K., Shetty, H. M., Paramel, A., \& Sharma, G. (2012). A retrospective study of extrapyramidal syndromes with second generation antipsychotics in the psychiatric unit of a tertiary care teaching hospital. Journal of pharmacology \& pharmacotherapeutics, 3(3), 266. DOI 10.4103/0976-500X.99435.

Laursen, T. M., Munk-Olsen, T., \& Vestergaard, M. (2012). Life expectancy and cardiovascular mortality in persons with schizophrenia. Current opinion in psychiatry, 25(2), 83-88. DOI 10.1097/YCO.0b013e32835035ca.

Lima, M. S. D., Soares, B. G. D. O., Paoliello, G., Vieira, R. M., Martins, C. M., Mota Neto, J. I. D., ... \& Volpe, F. M. (2007). The Portuguese version of the clinical global impressionschizophrenia scale: validation study. Revista brasileira de psiquiatria, 29(3), 246-249. DOI 10.1590/S1516-44462007000300010.

Lindner, L. M., Marasciulo, A. C., Farias, M. R., \& Grohs, G. E. M. (2009). Avaliação econômica do tratamento da esquizofrenia com antipsicóticos no Sistema Único de Saúde. Revista de Saúde Pública, 43(suppl. 1), 62-69. DOI 10.1590/S003489102009000800010.

Miasso, A. I., Miamoto, C. S., do Carmo Mercedes, B. P., \& Vedana, K. G. G. Adesão, conhecimento e dificuldades relacionados ao tratamento farmacológico entre pessoas com esquizofrenia. Revista Eletrônica de Enfermagem, 17(2), 186-95.

Nascimento, R. C., Lomez, E. D. S. L., \& Gaspar, J. (2014). Revisão sistemática para avaliar a eficácia do uso da Risperidona no tratamento de manutenção da esquizofrenia.

Oliveira, I.R. (2000). Antipsicóticos atípicos: Antipsicóticos atípicos: Antipsicóticos atípicos: farmacologia e uso clínico farmacologia e uso clínico. Revista Brasileira de Psiquiatria, 22(Supl I), 38-40, DOI 10.1590/S1516-44462000000500013.

Rang, H. P., Dale, M. M., Ritter, J. M., Flower, R. J., \& Henderson, G. (2012). O Sistema Vascular. Rang \&Dale Farmacologia. $7^{a}$ Edição. Elsevier, Rio de Janeiro, 265-284.

Rodrigues, L.S.M., Santos, A.S., Guerra Júnior, A.A., Alemão, M.M., Oliveira, H.N., Reis, E.A., Vidal, C.E.L., Brandão, C.M.R (2016). Hospitalização de pacientes esquizofrênicos no Sistema Único de Saúde em Minas Gerais, Brasil. Bras Econ Saúde, 8(3), 197-203.

Sales, C. F. N., \& Monteiro, K. M. S. L. (2016). Esquizofrenia e seus fatores adoecedores: um estudo multifatorial. Psicólogo inFormação, 19(19), 45-62. DOI 10.15603/21760969/pi.v19n19p45-62. 
Silva, G. D. D., Guerra Júnior, A. A., Acúrcio, F. D. A., Cherchiglia, M. L., \& Andrade, E. I. G. (2011). Medicamentos excepcionais para doença renal crônica: gastos e perfil de utilização em Minas Gerais, Brasil. Cad. saúde pública,27(2), 357-368. DOI 10.1590/S0102-311X2011000200017.

WHO. World Health Organization (2017). Mental Health. Disponível em: http://www.who.int/mental_health/management/schizophrenia/en/. Acesso em: 12 jun. 2017.

WHO. World Health Organization (1997). CID-10 Classificação Estatística Internacional de Doenças e Problemas Relacionados à Saúde. 10a rev. São Paulo: Universidade de São Paulo; 1997. vol.2.

Zago, A. C., Tomasi, E., \& Demori, C. C. (2015). Adherence to drug treatment regarding the users of psychosocial attention centers with mood disorders and schizophrenia. SMAD. Revista eletrônica saúde mental álcool e drogas, 11(4), 224-233.DOI 10.11606/issn.18066976.v11i4p224-233.

\section{As autoras:}

Aline Ferreira Campos Correia é farmacêutica, mestranda no Programa de Mestrado em Gestão Organizacional, Universidade Federal de Goiás (UFG), Regional Catalão. Catalão- GO, Brasil, e-mail: aline.farma.bio@hotmail.com

Ivânia Vera é docente da Universidade Federal de Goiás (UFG), Regional Catalão. Catalão - GO, Brasil, email: ivaniavera@gmail.com

Roselma Lucchese é docente da Universidade Federal de Goiás (UFG), Regional Catalão. Catalão - GO, Brasil, e-mail:roselmalucchese@hotmail.com

Kamylla Guedes de Sena é enfermeira, Mestranda no Programa de Mestrado em Gestão Organizacional, Universidade Federal de Goiás (UFG), Regional Catalão. Catalão- GO, Brasil, e-mail: kamylla_g.s@hotmail.com

Roberta Almeida Elias é tecnologia da Informação, Mestranda no Programa de Mestrado em Gestão Organizacional, Universidade Federal de Goiás (UFG), Regional Catalão. Catalão- GO, Brasil, e-mail: robertaellias@gmail.com

Recebido em: 21/01/2018.

Aprovado em: 26/05/2018 